\title{
Prevalence of Antibiotic Resistance to Isonicotinylhydrazide and Rifampicin in Culture Positive Pulmonary Tuberculosis Patients From 2014 to 2016 in Zahedan City, Iran
}

\author{
Seyed Mohammad Hashemi Shahri ${ }^{*}$, Fatemeh Fardoust ${ }^{1}$, Shokoufeh Mogharabi Ostad Kalayeh ${ }^{2}$, \\ Mohammad Ghenaatpisheh Sanani ${ }^{3}$
}

'Infectious Diseases and Tropical Medicine Research Center, Zahedan University of Medical Sciences, Zahedan, Iran ${ }^{2}$ Faculty of Nursing, Islamic Azad University, Iranshahr Branch, Iranshahr, Iran

${ }^{3}$ Faculty of Medical Sciences, Zahedan Branch, Islamic Azad University, Zahedan, Iran

*Corresponding Author: Seyed Mohammad Hashemi-Shahri, Infectious Diseases and Tropical Medicine Research

Center, Zahedan University of Medical Sciences, Zahedan, Iran. Email: dr.sm.hashemishahri@gmail.com

Received January 5, 2019; Accepted May 12, 2019; Online Published June 17, 2019

\begin{abstract}
Background: One third of the world's population is infected with TB, and the disease is known as the second deadly global infection, even more severe than measles. The disease kills about 2 million people a year. Approximately 8 million people in the world are affected by advanced tuberculosis (TB).

Objective: The current study aimed to determine the frequency of resistance to the antibiotics isoniazid and rifampin in positive culture pulmonary TB patients in Zahedan city during 2013-2016.

Methods: This descriptive cross-sectional study studied 100 samples from patients who referred to Boo-Ali hospital of Zahedan city from 2014 to 2016 . First, the isoniazid solution was prepared, and then the rifampin solution was prepared. After that, $200 \mu \mathrm{L}$ of mycobacterium suspension was added to the antibiotic and antibiotic-free control.

Results: Of 100 patients participating in the study, $50(50 \%)$ were male and $50(50 \%)$ were female. Participants had an average age of $51.69 \pm 20.41$ years (95-12 years), 79 (79\%) were Iranians and 21 (21\%) were Afghans. Of the 100 samples, 55 (55\%) were from the year 2013, 21 (21\%) were from 2014, $10(10 \%)$ were from 2015, and $14(14 \%)$ were from year 2016. The samples examined showed that $4(4 \%)$ were resistant and $96 \%$ were sensitive.

Conclusion: The results of the current study showed that out of 100 samples, 4 cases (4\%) had resistance and $96 \%$ were sensitive; $2 \%$ were resistant to isoniazid, and $2 \%$ were resistant to isoniazid and rifampin.

Keywords: Pulmonary Tuberculosis, Drug Resistance, Tuberculosis (TB)
\end{abstract}

\section{Background}

One third of the world's population is infected with tuberculosis (TB), a disease known as the second most deadly global infection; it is even more severe than measles. The disease kills about 2 million people a year, and about 8 million people in the world are affected by advanced TB. ${ }^{1}$ TB is caused by a bacterial agent called Mycobacterium tuberculosis. These bacteria can also cause diseases similar to other mycobacteria, such as Mycobacterium bovis. ${ }^{2-4}$ Mycobacterium tuberculosis causes tissue damage, and it can cause pneumonia in the lung. The disease may progress quickly and cause a lesion with an uncertain range of varying sizes and densities. This lesion is usually homogeneous if it is small and if it comes under the influence of anti-TB drugs. If the disease has not progressed to caseous necrosis, full recovery can be achieved. ${ }^{5}$ More than $90 \%$ of cases are found in developing countries, with $75 \%$ of those being patients between 15 and 54 years of age. In these countries, a person with $\mathrm{TB}$ is not able to work for about 3 to 4 months and will lose $20 \%$ to $30 \%$ of his family's annual income; on average, 15 year goes away from his family's income. ${ }^{6}$

In the absence of effective interventions, an epidemic of TB can involve a community for many years. Since the mid20th century, the presence of anti-TB drugs has resulted in a significant increase in epidemics. In general, two main factors have led to an increase in the number of TB cases in the world. The first incidence was HIV infection which

Copyright (C 2019 The Author(s). This is an open-access article distributed under the terms of the Creative Commons Attribution License (http:// creativecommons.org/licenses/by/4.0), which permits unrestricted use, distribution, and reproduction in any medium, provided the original work is properly cited. 
resulted in the destruction of two cells that play a major role in the fight against Mycobacterium (macrophages and CD4 lymphocytes). The second incidence was the emergence of resistance to anti-TB drugs. ${ }^{7}$ Drug resistance in a patient with $\mathrm{TB}$ occurs when the patient consumes one or more first-line drugs for the treatment of TB (isoniazid, rifampin, pyrazinamide, ethambutol, or streptomycin). First-line drugs are most effective in the treatment of TB, and all of them, with the exception of low-dose ethambutol, have bactericidal properties. The drug resistance in TB based on epidemiology is divided into 3 broad categories:

Primary: Patients who have not previously had any history of TB drug consumption yet now have drugresistant organisms that are contaminated by foreignsource resistant organisms. ${ }^{8}$

Acquired: Patients who initially had drug-susceptible organisms, but were then resistant to organisms due to inappropriate, inadequate, irregular, or, most importantly, non-compliance with treatment protocols. ${ }^{8}$

Initial: Patients who deny previous use of drugs and do not have a clear history of anti-TB drug consumption. ${ }^{8}$

\section{Objective}

Due to the high prevalence of TB in Iran and the absence of a detailed study on antibiotic resistance, and also considering the contradictory results in this regard, the current study aimed to determine the frequency of antibiotic resistance to isoniazid and rifampin antibiotics in positive culture pulmonary $\mathrm{TB}$ patients in Zahedan city during 2013-2016, thus taking an important step in identifying the antibiotic resistance of TB and changing patients' therapeutic policies.

\section{Methods}

This descriptive cross-sectional study evaluated all patients with positive sputum culture who referred to Boo-Ali hospital of Zahedan city from 2014 to 2016. In this study, all available live samples collected between 2013 and 2016 as well as all patients who had a positive sputum culture in 2016 were evaluated for resistance to isoniazid and rifampin. Approximately 100 samples were taken.

First, the isoniazid solution was prepared by dissolving 50 $\mathrm{mg}$ of isoniazid in $25 \mathrm{ml}$ distilled water. The stock solution contained $2000 \mu \mathrm{g} / \mathrm{mL}$. Next, $1 \mathrm{~mL}$ of stock solution was added to $9 \mathrm{ml}$ of distilled water. The solution contained 200 $\mathrm{mL} / \mu \mathrm{g}$ and was called solution $\mathrm{A}$. Then, $1 \mathrm{~mL}$ of solution $\mathrm{A}$ was added to $9 \mathrm{~mL}$ of distilled water, creating solution $\mathrm{B}(20$ $\mathrm{mL} / \mu \mathrm{g})$. The B solution was sterilized using a membrane filter, and then $1 \mathrm{ml}$ of filtered solution B was added to $99 \mathrm{cc}$ of Löwenstein-Jensen culture medium. The concentration of isoniazid in $1 \mathrm{ml}$ of LJ solution was $0.2 \mu \mathrm{g} / \mathrm{mL}$. Then, the rifampin solution was prepared by dissolving $40 \mathrm{mg}$ of rifampin in $10 \mathrm{cc}$ DMSO (stock solution containing 4000 $\mu \mathrm{g} / \mathrm{mL}$ rifampicin). The stock solution was sterilized with membrane filtering, and then $1 \mathrm{ml}$ of filtered solution was added to $99 \mathrm{cc}$ LJ. The rifampin concentration was $40 \mu \mathrm{g} /$ $\mathrm{mL}$. Then, 8-6 $\mathrm{mL}$ of the pharmaceutical compositions prepared in sterile tubes were added and cooled for 45 minutes at $85^{\circ} \mathrm{C}$ and stored at room temperature in a refrigerator for up to 1 month.

The McFarland technique, which shows the number of bacteria in one $\mathrm{cc}$ of suspension, was used for standardization. McFarland is made up of $0.1 \mathrm{~mL}$ of barium chloride $1.175 \%$ with $0.9 \mathrm{cc}$ sulfuric acid $1 \%$ and is equal to $108 \times 3$ bacteria. There are 8 to 10 pearls in a small wrapped glass. Then, $20 \mathrm{~mL}$ of distilled water was added and the tubes were sterilized in an autoclave. Approximately 8-10 colonies were prepared for the suspension and dissolved in tubes for 4 to 5 minutes with a shaker. After the colonies became uniform, they were left stagnant for 45 minutes. Then they were removed from the suspension with the tip of the sampler and added to a tube containing one cc distilled water. Suspension was added to give an equivalent of one McFarland and Concentrations 2-10 and 4-10 were then made. Next, $200 \mu \mathrm{L}$ of mycobacterium suspension was added to the antibiotic and the antibiotic-free control.

The tubes were then shaken slowly to allow the suspension to make contact with the entire surface of the environment. After 28 to 42 days, the number of colonies on each environment was read, and the number of colonies in each dilution were counted. The number of colonies in the drug-free control was also counted, and the number was multiplied by 100 . A result equal to or less than 1 confirmed the sensitivity of the bacterium to the drug; other results indicated the bacteria was resistant to the drug. This ratio (the presence of the drug in a number of colonies and the absence of a drug in a number of colonies) is called the critical point of antibiotics.

For data analysis, descriptive statistics tables, frequencies, percentages, and charts were used. The data was entered into SPSS version 22 and analyzed. To compare the variables in both groups, the t-test, chi square, and Fisher exact test or Mann-Whitney U test were used.

\section{Results}

This study was performed on 100 subjects, of which 50 (50\%) were male and 50 (50\%) were female, with an average age of $51.69 \pm 20.41$ years (range $=12-95$ years). Of the 100 patients who were included in the study, 79 (79\%) were Iranians and 21 (21\%) were Afghans. From the samples, 55 (55\%) were from the year 2013, 21 (21\%) were from 2014, $10(10 \%)$ were from 2015 , and 14 (14\%) were from the year 2016. Four cases (4\%) were resistant and $96 \%$ were sensitive. The findings of this study are presented in the following tables.

As shown in Table 1, 2\% of patients were resistant to isoniazid, and $2 \%$ were resistant to both isoniazid and rifampin. Mean and standard deviation of age in TB patients based on antibiotic resistance is shown in Table 2 .

As shown in Table 3, the frequency of antibiotic resistance was not statistically significant $(P>0.05)$ based on patient gender.

Based on Table 4, the frequency of antibiotic resistance was not statistically significant $(P>0.05)$. The year-based 
Table 1. Frequency of Resistance in Patients

\begin{tabular}{lll}
\hline Resistance & No. & $\%$ \\
\hline Isoniazid resistance & 2 & 2 \\
Isoniazid and rifampin resistance & 2 & 2 \\
Sensitivity & 96 & 96 \\
\hline
\end{tabular}

Table 2. Mean and Standard Deviation of Age in TB Patients Based on Antibiotic Resistance

\begin{tabular}{llll}
\hline Group & Number & Mean & SD \\
\hline Sensitivity & 96 & 51.63 & 20.11 \\
Resistance & 4 & 53 & 30.64 \\
\hline
\end{tabular}

Table 3. Frequency of Antibiotic Resistance Based on Patient Gender

\begin{tabular}{llcccc}
\hline \multirow{2}{*}{ Gender } & & \multicolumn{3}{c}{ Resistance } & \multirow{2}{*}{ P Value } \\
\cline { 3 - 5 } & & No & Yes & All & \\
\hline \multirow{2}{*}{ Female } & No. & 49 & 1 & 50 & \\
& $\%$ & 98.0 & 2.0 & 100.0 & \\
\multirow{2}{*}{ Male } & No. & 47 & 3 & 50 & \multirow{2}{*}{$0.617^{*}$} \\
& $\%$ & 94.0 & 6.0 & 100.0 & \\
\multirow{2}{*}{ All } & No. & 96 & 4 & 100 & \\
& $\%$ & 96.0 & 4.0 & 100.0 & \\
\hline
\end{tabular}

*Fisher exact test was used.

Table 4. Frequency of Antibiotic Resistance Based on Patient Race

\begin{tabular}{llcccc}
\hline \multirow{2}{*}{ Race } & & \multicolumn{3}{c}{ Resistance } & \multirow{2}{*}{ P Value } \\
\cline { 3 - 5 } & & No & Yes & All & \\
\hline \multirow{2}{*}{ Iranian } & No. & 76 & 3 & 79 & \\
& $\%$ & 96.2 & 3.8 & 100.0 & \\
\multirow{2}{*}{ Non-Iranian } & No. & 20 & 1 & 21 & \multirow{2}{*}{$0.841^{*}$} \\
& $\%$ & 95.2 & 4.8 & 100.0 & \\
\multirow{3}{*}{ All } & No. & 96 & 4 & 100 & \\
& $\%$ & 96.0 & 4.0 & 100.0 & \\
\hline
\end{tabular}

*Fisher exact test was used.

frequency of antibiotic resistance in this study is shown in Table 5.

\section{Discussion}

The current study purposed to determine the prevalence of resistance to the antibiotics isoniazid and rifampin in patients with pulmonary TB in Zahedan during 20132016. This study was performed on 100 subjects, of whom $50(50 \%)$ were male and $50(50 \%)$ were female, with an average age of $51.66 \pm 20.41$ years (range $=12-95$ years) Of the 100 study participants, 79 (79\%) were Iranians and 21 (21\%) were Afghans; 55 (55\%) cases were from the year 2013, 21 (21\%) were from 2014, 10 (10\%) were from 2015, and $14(14 \%)$ were from the year 2016. Of the 100 samples examined, 4 (4\%) were resistant and 96\% were sensitive; $2 \%$ had a resistance to isoniazid, and $2 \%$ had a resistance to both isoniazid and rifampin. The prevalence of resistance
Table 5. Frequency of Antibiotic Resistance Based on the Year Studied

\begin{tabular}{llccc}
\hline \multirow{2}{*}{ Year } & & \multicolumn{3}{c}{ Resistance } \\
\cline { 3 - 5 } & & No & Yes & All \\
\hline \multirow{2}{*}{2013} & No. & 52 & 3 & 55 \\
& $\%$ & 94.5 & 5.5 & 100.0 \\
\multirow{2}{*}{2014} & No. & 20 & 1 & 21 \\
& $\%$ & 95.2 & 4.8 & 100.0 \\
& No. & 10 & 0 & 10 \\
\multirow{2}{*}{2016} & $\%$ & 100.0 & 0 & 100.0 \\
& No. & 14 & 0 & 14 \\
\multirow{2}{*}{ All } & $\%$ & 100.0 & 0 & 100.0 \\
& No. & 96 & 4 & 100 \\
\hline
\end{tabular}

based on age, gender, race, and year showed no significant difference.

In a study conducted by Moghtaderi et al on 235 suspected drug resistant patients in Tabriz, 96\% of the samples were resistant to streptomycin, $89 \%$ to isoniazid, $79 \%$ to rifampin, and $79 \%$ to etambutolum. ${ }^{9}$ In the current study, only $4 \%$ had a resistance to the antibiotics. The reasons for the different results are differences in sample sizes, demographic characteristics of the individuals, the criteria for entering and leaving the study, and in the control of the effects of the confounders.

In a study by Fasih et al in 2009-2011 on $7738 \mathrm{M}$. tuberculosis lung specimens in Pakistan, cultured in the Löwenstein-Jensen medium, it was found that the concurrent resistance to isoniazid, pyrazinamide, and ethambutol was $1 \%$, while resistance to isoniazid, pyrazinamide, ethambutol, and streptomycin was $1.7 \% .^{10}$ The results of this study are similar to those of the current study, in which it was also found that co-levels of isoniazid and rifampin were $2 \%$. In a study carried out by Tavanai Sani et al in 2012, 125 samples of positive TB in Mashhad were cultured and antibiogrammed. It was determined that resistance to isoniazid was $7 \%$, to rifampin was $7 \%$, to ethambutol was $3.9 \%$, to streptomycin was $11.8 \%$, and to isoniazid and rifampin was $4 \%{ }^{11}$ The results of this study are similar to those of the current study in which it was also found that co-levels of isoniazid and rifampin were $2 \%$. For each antibiotic, however, the resistance was significantly higher than in the current study. The reasons for this difference are differences in sample size, the demographic characteristics of individuals, the criteria for entering and leaving the study, and in the control of the effects of the confounders.

In a study by Farazi et al conducted from 2005 to 2010 on 917 patients with pulmonary TB and antibiograms, it was found that $7.3 \%$ of the patients had a positive sputum smear with a drug resistance of $3.4 \%$ to multi-drugresistant (MDR), and $0.5 \%, 68.8 \%, 62.5 \%, 25 \%, 21 \%$, and $9.21 \%$ of these people were resistant to all 5 first-line drugs, isoniazid, rifampin, pyrazinamide, ethambutol, and 
streptomycin, respectively. ${ }^{12}$ In the current study, only $4 \%$ had antibiotic resistance. This difference can be explained by differences in sample size, demographic characteristics of the individuals, the criteria for entering and leaving the study, and in the control of the effects of the confounders.

Das et al studied 850 suspected pulmonary TB patients in the Löwenstein-Jensen medium and found that 161 patients had pulmonary TB. The prevalence of MDR in this group was $0.85 \%$. Patients without a history of treatment and $4.54 \%$ were among those with a previous history of treatment. Also, the resistance rates in the group not treated so far were $3.41 \%, 2.56 \%, 0.85 \%$, and $2.27 \%$ for streptomycin, isoniazid, rifampin, and ethambutol, respectively. ${ }^{13}$ The results of this study are in line with the findings of the current study.

Roshdi Maleki and Moaddab aimed to determine the drug sensitization of mycobacterium tuberculosis strains in comparison with first-line and alternative drugs in Tabriz. First, using biochemical tests, 103 strains of MTB were isolated from samples from native patients referring to the Tuberculosis Research Center of Tabriz. After preparing the suspension, they were cultured on LöwensteinJensen medium with first- and second-line therapies. The results were analyzed using the proportional method. The standard strain of $\mathrm{H} 37 \mathrm{Rv} M$. tuberculosis, which is susceptible to all drugs, was used as a qualitative test. First-line drugs include isoniazid, rifampin, ethambutol, and streptomycin; second-line drugs include amikacin, kanamycin, ofloxacine, and ciprofloxacin. Of the 103 strains, 13 strains were resistant to kanamycine, 2 strains (1.9\%) to ofloxacin, 1 strain (1\%) to amikacin, and 1 strain (1\%) to ciprofloxacin. The greatest drug resistance for first-line drugs was seen for streptomycin, occurring in $7.8 \%$ (8 strains). Among the second-line drugs, the greatest drug resistance was seen for kanamycin (12.6\%) (13 strains). MDR-TB resistance was 2.9\% (3 strains). The vast majority of strains were sensitive to all first-line antiTB drugs and low MDR-TB rates. Among the main and alternative anti-TB drugs, streptomycin and kanamycin, respectively, showed the highest drug resistance. ${ }^{14}$ The study results on the low incidence of drug resistance to isoniazid and rifampin are consistent with the findings of the current study.

Pourhajibagher et al purposed to determine the resistance of mycobacterium tuberculosis to the drugs isoniazid and rifampin. This cross-sectional study was performed on 1345 patients who referred to the Mazandaran Tuberculosis Center in one year. Samples prepared from these individuals were cultured on LöwensteinJensen medium. DNA extraction from the colony and determination of resistance to isoniazid and rifampin by polymerase chain reaction (PCR) were performed using the primers of $M U T B-g y r B$, inhA, katG, and $r p o B$ genes, respectively. From 1345 samples, 65 positive culture samples were obtained. Using MUTB-gyrB gene primers, 59 of these 65 samples were determined to be $M$. tuberculosis. Resistance to isoniazid was detected by the
inhA gene primer in 3 cases (5.08\%), and sensitivity was detected in 56 cases $(94.92 \%)$; by the kat $G$ gene primer, resistance was detected in 4 cases $(6.77 \%)$ and sensitivity in 55 cases $(93.23 \%)$. Rifampin resistance was detected by rpoB primer in 1 case $(1.7 \%)$, and sensitivity was detected in 58 cases $(98.3 \%)$. The highest rate of resistance was that against isoniazid. PCR was shown to be a suitable method for identifying strains resistant and susceptible to isoniazid and rifampin..$^{15}$ Although the prevalence of resistance in Pourhajibagher et al's ${ }^{15}$ study was higher than that found in the current study, their results are consistent with the current findings.

Tayeboon et al aimed to diagnosis pulmonary TB and simultaneously determine drug resistance to isoniazid in clinical strains of $M$. tuberculosis using the PCR-restriction fragment length polymorphism (RFLP) method by studying 87 clinical samples of $M$. tuberculosis. At first, with the help of PCR, kat $G$ gene replication was performed on bp620 for molecular confirmation in bacterial diagnosis. The PCR product was then cut off using RFLP and appropriate enzymes, and the patterns obtained from electrophoresis were investigated to detect mutations in $315 \mathrm{katG}$. To confirm the final results, a number of samples were sequenced. All the studied strains showed a bp620 band that confirmed Mycobacterium tuberculosis. In addition, 46 strains resistant to isoniazid were shown by the RFLPPCR method, 44 of which had mutations in Ser315Thr of the kat $G$ gene. Of the 41 susceptible strains, none were mutated in codon $315 \mathrm{katG}$. On the other hand, sequencing results were used to validate the determination of the mutation using a molecular technique. In this research, the sensitivity rates of RFLP-PCR were calculated to be $95.6 \%$ (95\% CI: 0.85-0.98\%) and 100\% (0.91-0.9-1.95 CI).

The results of this study showed that the PCR-RFLP method can detect resistance to isoniazid in $95 \%$ of cases and can be used simultaneously to detect pulmonary TB and to determine drug resistance to isoniazid in clinical strains of Mycobacterium tuberculosis. Although the prevalence of resistance in Tayeboon et al's ${ }^{16}$ study was higher than that in the current study, their results are consistent with the current findings.

\section{Conclusion}

Results of the current study showed that out of 100 samples, 4 cases (4\%) were resistant and $96 \%$ were susceptible. Moreover, $2 \%$ had a resistance to isoniazid, and $2 \%$ had a resistance to isoniazid and rifampin. No significant difference in the prevalence of resistance was found based on age, gender, or race. Although the rate of resistance to isoniazid and rifampin in the current study was lower than that in other studies, due to the number of cases of MDR TB in Zahedan, the DOTS strategy should be fully and properly implemented to prevent the occurrence of most resistant cases.

\section{Limitation}

The current study was limited by a failure to cooperate 
with patients, which may have been somewhat resolved by further explaining the study and expressing its significance.

\section{Authors' Contributions}

SMHS contributed to conceptualization and data collecting; FF contributed to manuscript preparation; SMOK contributed to manuscript revising; MGS contributed to manuscript preparation and submission; SMHS participated in data analysis.

\section{Conflict of Interest Disclosures}

The authors declare that they have no conflicts of interest.

\section{Ethical Approval}

This study was approved by the Zahedan University of Medical Sciences Research Committee and was given code IR.ZAUMS.REC.1396.112.

\section{References}

1. Andreu J, Caceres J, Pallisa E, Martinez-Rodriguez M. Radiological manifestations of pulmonary tuberculosis. Eur J Radiol. 2004;51(2):139-149. doi:10.1016/j.ejrad.2004.03.009.

2. Sharifi MR, Forat Yazdi M, Amooghadiri M, Heidari H, Shaker M, Zeinolabedin M. Study of Diseases Associated ESR above 100 among the Patients who Visited Shahid Sadoughi Hospital in the Second half of 2013. Intern Med Med Investig J. 2006;1(1):5-9. doi:10.24200/imminv.v1i1.12.

3. Kim WS, Choi JI, Cheon JE, Kim IO, Yeon KM, Lee HJ. Pulmonary tuberculosis in infants: radiographic and CT findings. AJR Am J Roentgenol . 2006;187:1024-33.

4. Buckner CB, Leithiser RE, Walker CW, Allison JW. The changing epidemiology of tuberculosis and other mycobacterial infections in the United States: implications for the radiologist. AJR Am J Roentgenol. 1991;156(2):255-264. doi:10.2214/ ajr.156.2.1898796.

5. FitzGerald JM, Grzybowski S, Allen EA. The impact of human immunodeficiency virus infection on tuberculosis and its control. Chest. 1991;100(1):191-200. doi:10.1378/ chest.100.1.191.

6. Cremin BJ. Tuberculosis: the resurgence of our most lethal infectious disease--a review. Pediatr Radiol. 1995;25(8):620626. doi:10.1007/bf02011832.

7. Abbasi F, Yousefi S, Afshar Yavari S. Minimum inhibitory concentration of ciprofloxacin against $P$ seudomonas aeruginosa in the presence of the efflux inhibitor phenylalanine-arginine beta-naphthylamide. Intern Med Med Investig J. 2018;3(4):2327. doi:10.24200/imminv.v2i4.128.

8. Gangadharam PRJ. Drug resistance in tuberculosis. In: Reichman LB, Hershfield ES, eds. Tuberculosis: A Comprehensive International Approach. New York: Marcel

\section{Research Highlights}

\section{What Is Already Known?}

Recent studies have shown that isoniazid and rifampicin resistance have been presumed to have minimal clinical impact, causing it to be a topic of debate. One study showed an association between isoniazid and rifampin resistance and treatment failure.

\section{What This Study Adds?}

In the current study, out of 100 samples, 4 cases (4\%) had resistance and $96 \%$ were sensitive; $2 \%$ were resistant to isoniazid, and $2 \%$ were resistant to isoniazid and rifampin. These results will help future studies and scientists consider the resistance of these 2 drugs.

Dekker; 1993:293-328.

9. 9. Moghtadery R, Moaddab R, Rafi AN. Resistance of atypical Mycobacterium as pulmonary infections agents to the first and second line of anti tuberculosis drugs. Iranian Journal of Infectious Diseases and Tropical Medicine. 2012;17(58):59-63.

10. Fasih N, Rafiq Y, Jabeen K, Hasan R. High isoniazid resistance rates in rifampicin susceptible Mycobacterium tuberculosis pulmonary isolates from Pakistan. PLoS One. 2012;7(11):e50551. doi:10.1371/journal.pone.0050551.

11. Tavanai Sani A, Ghazvini K, Salehi M, Shakiba A. Investigating the Resistance of Mycobacterium tuberculosis to anti-TB drugs and its effective factors in the samples referred to Shariati Hospital of Mashhad. Journal of Faculty of Medicine, Mashhad University of Medical Sciences. 2015;57(8):898-903. [Persian].

12. Farazi A, Jabbariasl M, Sofian M. Assessment of drug resistance in tuberculosis patients and the factors affecting it (2005-2010). Arak Medical University Journal. 2012;15(60):77-85.

13. Das D, Satapathy P, Murmu B. First Line Anti-TB Drug Resistance in an Urban Area of Odisha, India. J Clin Diagn Res. 2016;10(11):Dc04-dc06. doi:10.7860/jcdr/2016/20289.8846.

14. Roshdi Maleki M, Moaddab SR. Drug susceptibility pattern of Mycobacterium tuberculosis strains to first and second line drugs in Tabriz, Iran. Iran J Med Microbiol. 2009;3(1):18-24.

15. Pourhajibagher M, Nasrollahi M, Musavi S, Rahimi-Esboei B, Ghorbani Pashakolaei A. Drug resistance in Mycobacterium tuberculosis isolates to isoniazid and rifampin. Journal of Babol University of Medical Sciences. 2012;14(3):66-72.

16. Tayeboon MA, Sadrnia M, Mohajerani H. Simultaneous detection of TB and drug resistance to Isoniazid in Mycobacterium tuberculosis clinical isolates using PCR-RFLP method. Iran South Med J. 2015;18(3):547-555. 